\title{
Diagnostic significance of a color Doppler ultrasound combined with serum CXCL16 and E-cad in cervical cancer
}

\author{
Wenrong Wang ${ }^{1}$, Xing Gao ${ }^{1}$, Yuan Zhu ${ }^{1}$, Yaozhi Qi ${ }^{2}$, Yanjuan Wang ${ }^{3}$ \\ ${ }^{1}$ Department of Ultrasound, Lianyungang Maternal and Child Health Hospital, Lianyungang, China; ${ }^{2}$ Department of Laboratory, Lianyungang \\ Maternal and Child Health Hospital, Lianyungang, China; ${ }^{3}$ Department of Child Psychology, Lianyungang Maternal and Child Health Hospital, \\ Lianyungang, China \\ Contributions: (I) Conception and design: W Wang; (II) Administrative support: Y Wang, Y Qi; (III) Provision of study materials or patient data: Y \\ Zhu; (IV) Collection and assembly of data: X Gao; (V) Data analysis and interpretation: W Wang; (VI) Manuscript writing: All authors; (VII) Final \\ approval of manuscript: All authors. \\ Correspondence to: Yanjuan Wang; Yaozhi Qi. Lianyungang Maternal and Child Health Hospital, Lianyungang, China. \\ Email: 474988527@qq.com; 372055430@qq.com.
}

Background: The use of effective non-invasive diagnostic markers in the early stage of cervical cancer is lacking currently. This study sought to investigate the value of color Doppler ultrasound combined with serum CXC chemokine ligand-16 (CXCL16) and epithelial cadherin (E-cad) levels in diagnosing cervical cancer.

Methods: Two hundreds cervical cancer patients admitted in our hospital between May 2018 and April 2020 were selected as the observation group. The control group included 100 healthy participants. The ultrasound parameters, and the serum CXCL16 and E-cad levels between the two groups and patients at different pathological stages were compared. The correlations between disease progress and ultrasound and serological indexes were analyzed. The diagnostic efficiency of an ultrasound and measures of serum CXCL16 and E-cad levels were compared as well.

Results: The peak systolic velocity (PSV) of the observation group was significantly higher than that of the control group $(\mathrm{P}<0.05)$. In case of the resistance index $(\mathrm{RI})$, the results were the opposite to the PSV $(\mathrm{P}<0.05)$. The PSV $(\mathrm{P}<0.05)$ and $\mathrm{RI}(\mathrm{P}<0.05)$ were significantly different among different pathological stages. The serum CXCL16 $(\mathrm{P}<0.05)$ and E-cad $(\mathrm{P}<0.05)$ levels of participants in the observation group were significantly higher than those of patients in the control group. A pairwise comparison showed that the serum CXCL16 and E-cad levels increased significantly as the pathological stages of the cancer progressed. The diagnosis and disease progression of cervical cancer were positively correlated with PSV, CXCL16, and E-cad levels, and negatively correlated with the RI. Combining an ultrasound diagnosis with serum CXCL16 and E-cad levels had significantly higher diagnostic sensitivity than that of any individual indicator in patients with cervical cancer. The curve analysis showed that the cut-off values of PSV, the RI, CXCL16 and E-cad were $14.25,0.50,40.15 \mathrm{ng} / \mathrm{mL}$ and $85.36 \mathrm{pmmol} / \mathrm{L}$, respectively.

Conclusions: The sensitivity of color Doppler ultrasound combined with serum CXCL16 and E-cad levels in the diagnosis of cervical cancer is high. Thus, it is recommended that it be used in clinics.

Keywords: Color Doppler ultrasound; peak systolic velocity (PSV); resistance index (RI); CXC chemokine ligand-16 (CXCL16); E-cad; cervical cancer

Submitted Dec 07, 2020. Accepted for publication Mar 05, 2021.

doi: $10.21037 /$ tcr-21-123

View this article at: http://dx.doi.org/10.21037/tcr-21-123 


\section{Introduction}

The incidence of cervical cancer is ranked second among female malignant tumors in the world (1), and its age of onset has a significant downward trend in epidemiological investigations, which seriously affects the life safety of patients. Additionally, the clinical symptoms of cervical cancer are not obvious, such that by the time of diagnosis, the vast majority of patients have progressed into the middle or late stages of cervical cancer. Thus, the timely diagnosis of patients at an early stage has significant effects on the prognosis of patients (2). In the process of tumor progression, most of the neovascularization are flexuous, and the deformity rate is high due to the significant imbalance of vascular endothelial cell function. Consequently, the maximum peak systolic velocity (PSV) and resistance index (RI) (3) of the hemodynamic arteries around the mass and the mass examined by transvaginal color Doppler ultrasound can reflect the angiogenesis of the tumor to some extent. Further, ultrasounds show the progress of tumors; however, the diagnostic efficiency of ultrasounds is low. Thus, it is important that biomarkers be identified in a timely manner in clinical diagnoses.

Many studies have explored the correlation between biomarkers and the results of transvaginal color Doppler ultrasonography for cervical cancer, such as $\mathrm{p} 53$. However, the aim of the study was to assess the effectiveness of radiotherapy (RT) by detecting the expression of $\mathrm{p} 53$ protein combined with the signs of transvaginal color Doppler ultrasound in patients with advanced cervical cancer (4). The biomarkers used to diagnose cervical cancer are carcinoembryonic antigen (CEA) and squamous cell carcinoma antigen (SCC). Carcinoembryonic antigen is a commonly used biomarker of cervical cancer. However, abnormally elevated expression of CEA can also be seen in cancers such as rectal cancer, breast cancer, and lung cancer (5-7). Therefore, the specificity for the diagnosis of cervical cancer is poor. SCC is a specific antigen secreted by squamous cell carcinoma, which is related to the development of cervical cancer. It has high specificity but low sensitivity (8). Studies have found that chemokine 16 (chemokine 16, CXCL16) is related to tumor metastasis, invasion, adhesion and directional migration. The studies had confirmed that serum CXCL16 expression had the highest positive rate in cervical cancer. Among other diseases of the cervix, the positive rate is low and the specificity is high (9).

E-cad, a calcium-dependent cell adhesion protein that help to enhance the adherens junctions between cells, that is significantly correlated with tumor migration, invasion, and prognosis $(10,11)$. CXCL16, a cxcr6 receptor-binding chemokine that functions as a scavenger receptor for oxidized low density lipoprotein (oxldl) when expressed by macrophages (12) are the most promising biomarkers for the early diagnosis of cervical cancer. Trend factor interleukin-16, Interleukin-8, and CXC chemokine ligand-16 (CXCL16) reflect the levels of inflammatory responses in local lesions to some extent (13). The decrease of E-cad expression will increase the risk of tumor invasion, early occurrence and metastasis. Five hundred and twentytwo $(42.6 \%)$ participants included in 20 studies presented reduced E-cad expression. The downregulation of E-cad was associated with unfavorable overall survival in cervical cancer patients. E-cadmay can act as a metastatic suppressor protein, and therefore can be used as a diagnostic biomarker for cervical cancer. The increased expression of CXCL16 will increase the risk of tumor metastasis, invasion, adhesion and directional migration. The CXCL16 is present in the membrane and cytoplasm of neoplastic epithelial cells. While the tumor lesions progress from CIN1, CIN2, and CIN3 to invasive cancer, the distribution and expression intensity of CXCL16 increased as well. The expression of CXCL16 and CXCR6 in healthy cervical tissue, CIN, and cervical carcinoma were analyzed by using tissue chip technology and immunohistochemistry method. The association of protein expression with clinicopathologic features and overall survival was analyzed by using KaplanMeier curve. The patients with higher CXCR6 expression had significantly lower overall survival rates as compared to those with lower CXCR6 expression. The increased co-expression of CXCL16/CXCR6 in CIN and cervical cancer indicates that the development of cervical cancer is a durative process $(14,15)$. If the expression of E-cad decreases and the expression of CXCL16 increases, it is highly likely to predict the formation and migration of cervical cancer.

The objective of this study is to assess the diagnostic value of a color Doppler ultrasound combined with measures of serum CXCL16 and E-cad levels in the diagnosis of cervical cancer. We hypothesis that the diagnostic accuracy could be significantly improved by combining these 3 markers, compared with using single indicator. We present the following article in accordance with the STARD reporting checklist (available at http://dx.doi.org/10.21037/tcr-21-123). 


\section{Methods}

\section{Study design}

This is a retrospective study carried out in Lianyungang Maternal and Child Health Care Hospital in Jiangsu province China which was performed in accordance with the STARD 2015 guidelines for reporting diagnostic accuracy studies (16).

\section{General information}

200 patients, who had been diagnosed with cervical cancer and treated at our hospital from May 2018 to April 2020, participated in this prospective study. The patients were aged 40-49 years old, and had an average age of $45.57 \pm 2.09$ years and an average body mass index (BMI) of $24.25 \pm 2.18 \mathrm{~kg} / \mathrm{m}^{2}$. According to the pathological grading standard of the International Federation of Obstetrics and Gynecology of Cervical Cancer (FIGO), the average maximum diameter of a tumor is $19.90 \pm 3.01 \mathrm{~mm}(17)$. Of the patients, 45 had stage I cervical cancer, 92 had stage II cervical cancer, and 63 had stage III cervical cancer. One hundred healthy people from the same period were also selected to participate in the study as members of the control group. Notably, there were no significant differences in the general data between the two groups $(\mathrm{P}>0.05)$. All the patients signed the informed consent form, which was provided to and approved by the Ethics Committee of Lianyungang Maternal and Child Health Care Hospital (No.: LYG-ME2020056). All procedures performed in this study involving human participants were in accordance with the Declaration of Helsinki (as revised in 2013).

To be eligible to participate in the study, the patients had to meet the following inclusion criteria: (I) meet the diagnostic criteria for cervical cancer (6); (II) have no other primary tumors; and (III) have a card score of more than 60. Patients were excluded from the study if: (I) they had a mental disorder; (II) could not communicate normally; (III) were lactating or pregnant; and/or (IV) had other reproductive system diseases.

\section{Research methods}

\section{Ultrasonic diagnosis}

The ultrasonic instrument used in this study was a Siemens AcusonAntares ultrasonic diagnostic instrument. The frequency of the vaginal probe was set to $7.5 \mathrm{~m}$. The shape, size, edge, and internal shape of the uterus were diagnosed using a two-dimensional ultrasound. Additionally, the blood flow at the site of the lesions was compared using color Doppler flow imaging after the diagnoses had been made. The blood flow velocity related indexes of the three vessels were also measured, and the PSV and RI of the hemodynamic artery were recorded, and their average values were calculated in the area for which the ultrasound showed the most significant blood flow.

\section{Serological diagnosis}

Elbow vein blood was collected timely after enrollment and supernatant was collected after centrifugation at $3,500 \mathrm{r} / \mathrm{min}$ after all the patients had been enrolled in the group. Additionally, the CXCL16 and E-cad levels of the patients were analyzed using an enzyme-linked immunosorbent assay (Roche, Shanghai). The operation procedure was carried out in strict accordance with the instructions.

\section{Observation index}

* The ultrasonic parameters of the PSV and the RI of the observation group and the control group were compared.

* The ultrasonic parameters of the PSV and the RI of patients at different pathological (i.e., stages I, II, and III) were compared.

* The serum indexes (i.e., serum CXCL16 and E-cad levels) of the observation group and the control group were compared.

- The serum CXCL16 and E-cad levels in patients at different pathological stages (i.e., stages I, II, and III) were compared.

* The correlation between the disease progression and ultrasonic and serological indexes were analyzed. A Spearman correlation analysis was used to analyze the progress of the disease, and determine the correlations between disease diagnoses and ultrasound and serological indexes.

* The efficacy of an ultrasonic diagnosis combined with measures of serum CXCL16 and E-cad levels was analyzed. The receiver operating characteristic curve (ROC) was used to analyze the efficacy of the combined diagnosis and the individual diagnoses of the ultrasound and serum CXCL16 and E-cad levels. 


\section{Statistical analysis}

SPSS20.0 was used to collect and analyze the data. Additionally, a Chi-square test and an independent sample $t$-test were used to compare patients' basic data; a correlation analysis was used for the Spearman analysis, and diagnostic efficiency was used for the ROC curve analysis. The measurement data were expressed as mean \pm standard deviation (SD), and the count data was expressed as a percentage. $\mathrm{A} \mathrm{P}<0.05$ indicated that a difference was statistically significant.

\section{Results}

Comparison of ultrasonic parameters between the two participant groups

As Table 1 and Figure 1 shows, the PSV of the patients in the observation group was significantly higher than that of the PSV of patients in the control group ( $t=17.914$, $\mathrm{P}<0.05)$. The RI of patients the observation group was significantly lower than that of patients in the control

Table 1 Comparison of ultrasonic parameters between the two groups

\begin{tabular}{lccc}
\hline Group & Number of cases & PSV & $\mathrm{Rl}$ \\
\hline Control group & 100 & $10.15 \pm 3.11$ & $0.70 \pm 0.27$ \\
Observation & 200 & $16.59 \pm 2.55$ & $0.42 \pm 0.16$ \\
group & & & \\
$\mathrm{t}$ & 17.914 & 9.565 \\
$\mathrm{P}$ & 0.000 & 0.000 \\
\hline
\end{tabular}

PSV, peak systolic velocity; RI, resistance index. group $(\mathrm{t}=9.565, \mathrm{P}<0.05)$.

\section{Comparison of ultrasonic parameters in patients at different pathological stages}

As Table 2 shows, there were significant differences between the PSV $(\mathrm{F}=14.345, \mathrm{P}<0.05)$ and $\mathrm{RI}(\mathrm{F}=15.090, \mathrm{P}<0.05)$ of patients at different pathological stages. A pairwise comparison showed that PSV significantly increased and the RI significantly decreased as the pathological stage progressed.

\section{Comparison of serum indexes between the two groups of participants}

The serum CXCL16 and E-cad levels of participants in the observation group were significantly higher than those of participants in the control group (Table 3).

\section{Comparison of serum CXCL16 and E-cad levels in patients at different pathological stages}

There were significant differences in the serum CXCL16 and E-cad levels of patients at different pathological stages. A pairwise comparison showed that serum CXCL16 and E-cad levels increased significantly as the pathological stages progressed (see Table 4).

\section{Analysis of the correlation between disease progression and the ultrasonic and serological indexes}

The diagnosis and progression of cervical cancer were positively correlated with the levels of PSV, CXCL16 and

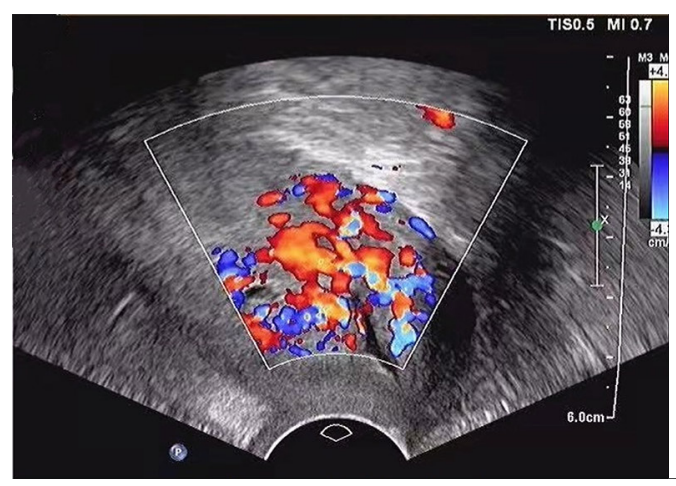

A color doppler flow imaging

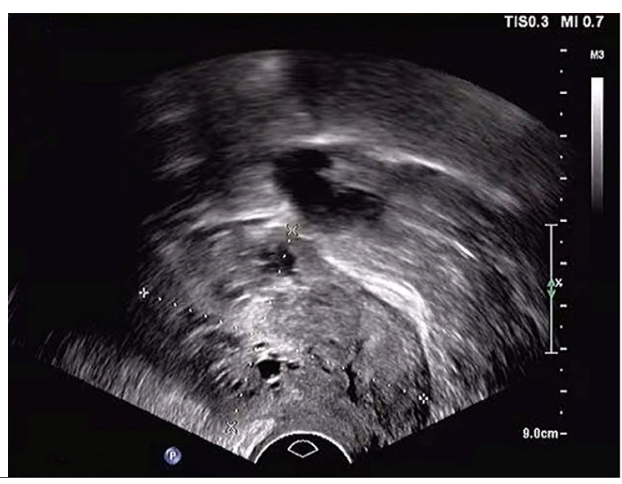

B doppler imaging

Figure 1 Ultrasonic images of patients in the observation group. 
Table 2 Comparison of ultrasonic parameters in patients at different pathological stages

\begin{tabular}{lccc}
\hline Group & $\mathrm{N}$ & $\mathrm{PSV}$ & $\mathrm{RI}$ \\
\hline I & 45 & $15.55 \pm 5.11$ & $0.51 \pm 0.22$ \\
II & 92 & $16.40 \pm 5.22^{\mathrm{a}}$ & $0.41 \pm 0.12^{\mathrm{a}}$ \\
III & 63 & $17.35 \pm 5.29^{\mathrm{ab}}$ & $0.10 \pm 0.10^{\mathrm{ab}}$ \\
F & & 14.345 & 15.090 \\
P & & 0.000 & 0.000 \\
\hline a , compared with stage I (P<0.05); ${ }^{\mathrm{b}}$, compared with stage II \\
(P<0.05). PSV, peak systolic velocity; RI, resistance index.
\end{tabular}

Table 3 Comparison of serum indexes between the two groups

\begin{tabular}{lccc}
\hline Group & $\mathrm{N}$ & $\mathrm{E}-\mathrm{cad}(\mathrm{ng} / \mathrm{mL})$ & $\mathrm{CXCL} 16(\mathrm{pmmol} / \mathrm{L})$ \\
\hline Control group & 100 & $16.25 \pm 3.52$ & $2.44 \pm 0.54$ \\
$\begin{array}{l}\text { Observation } \\
\text { group }\end{array}$ & 200 & $42.77 \pm 11.25$ & $92.33 \pm 10.16$ \\
$\mathrm{t}$ & & & \\
$\mathrm{P}$ & & 17.994 & 10.547 \\
\hline
\end{tabular}

CXCL16, CXC chemokine ligand-16.

Table 4 Comparison of serum CXCL16 and E-cad levels in patients at different pathological stages

\begin{tabular}{lccc}
\hline Group & $\mathrm{N}$ & E-cad $(\mathrm{ng} / \mathrm{mL})$ & CXCL16 $(\mathrm{pmmol} / \mathrm{L})$ \\
\hline I & 45 & $40.39 \pm 5.35$ & $85.79 \pm 3.21$ \\
II & 92 & $42.55 \pm 5.40^{\mathrm{a}}$ & $91.32 \pm 3.40^{\mathrm{a}}$ \\
III & 63 & $45.39 \pm 5.35^{\mathrm{ab}}$ & $97.79 \pm 3.21^{\mathrm{ab}}$ \\
F & & 16.541 & 18.217 \\
P & & 0.000 & 0.000 \\
\hline
\end{tabular}

${ }^{a}$, compared with stage I $(\mathrm{P}<0.05) ;{ }^{\mathrm{b}}$, compared with stage II $(\mathrm{P}<0.05)$. CXCL16, CXC chemokine ligand-16.

E-cad, and negatively correlated with the RI; the differences were statistically significant (see Table 5).

\section{Analysis of the efficacy of the ultrasonic diagnosis combined with serum CXCL16 and E-cad levels}

The sensitivity of the ultrasonic diagnosis and the combined diagnosis of serum CXCL16 and E-cad levels in patients with cervical cancer was significantly higher than that in patients with cervical cancer alone. The ROC curve showed
Table 5 Correlation analysis of disease progression with ultrasound and serological indexes

\begin{tabular}{lcccc}
\hline Index & PSV & RI & E-cad & CXCL16 \\
\hline Cervical cancer & & & & \\
r & 0.592 & 0.988 & 0.766 & 0.511 \\
P & 0.000 & 0.000 & 0.000 & 0.000 \\
$\begin{array}{l}\text { Staging of cervical } \\
\text { cancer }\end{array}$ & & & & \\
r & 0.902 & 0.641 & 0.823 & 0.561 \\
P & 0.000 & 0.000 & 0.000 & 0.000 \\
\hline
\end{tabular}

PSV, peak systolic velocity; RI, resistance index; CXCL16, CXC chemokine ligand-16.

that the cut-off values of PSV, RI, CXCL16, and E-cad in patients with cervical cancer were $14.25,0.50,40.15 \mathrm{ng} / \mathrm{mL}$, and $85.36 \mathrm{pmmol} / \mathrm{L}$, respectively (see Table 6 and Figure 2).

\section{Discussion}

As one of the most common gynecological malignant tumors in clinic, the medical imaging diagnosis of cervical cancer has made considerable progress due to the continuous improvement of clinical diagnostic skills (18). In the early stage of the disease and at the various stages of treatment, timely adjustment of the treatment protocols has positive significance for the patients (19). Further, there is a significant correlation between the migration of tumor cells and the formation of micro-vessels infiltrated in tumor cells in the study of the disease progression of cervical cancer (20). The objective index of tumor patients' infiltration and progression captures the increase of microvessel density in the local focus in the study of vascular endothelial cells of tumor cells (21). The probability of tumor cells entering the blood through microcirculation increases significantly (22), increasing the risk of distant spread of tumor.

The analysis of the ultrasonic indexes of the patients revealed that the PSV of patients in the observation group was significantly higher than that of the control group, and the RI of patients in the observation group was significantly lower than that of the control group. A pairwise comparison showed that the PSV of the patients increased significantly and the RI decreased significantly as the pathological stage of the patients progressed. The analysis showed that the measurements of PSV and RI 


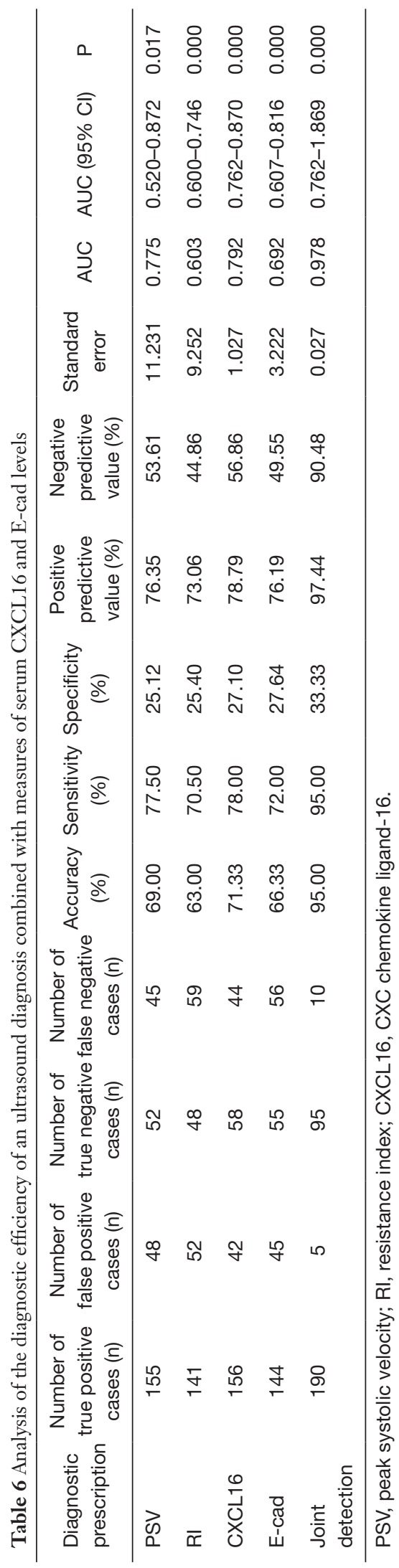

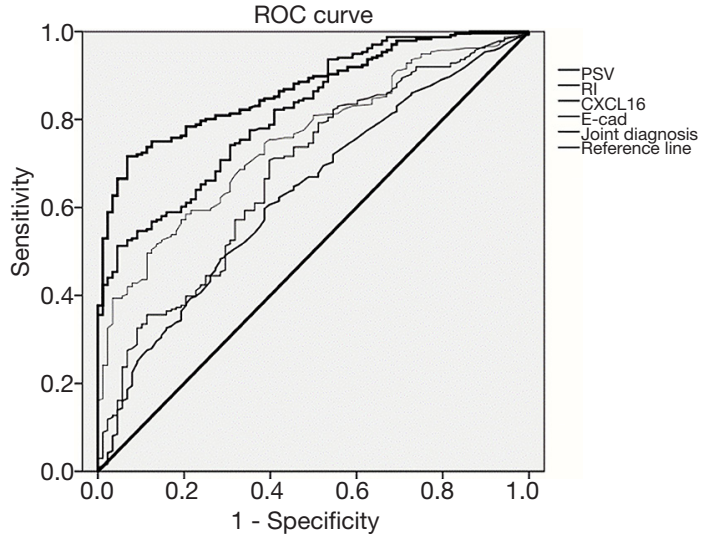

Figure 2 Analysis of diagnostic efficiency of the ultrasound combined with measures of serum CXCL16 and E-cad levels. CXCL16, CXC chemokine ligand-16.

mainly reflected the blood flow velocity and vascular elastic resistance. During the progression of the disease, due to the significant changes in metabolic function, the ability of tumor cells to plunder nutrients from the lesion site is significantly increased, which necessitate a large number of blood vessels to transport the nutrients. Moreover, in the process of angiogenesis, the malformation rate of neovascularization increases significantly due to a local inflammatory reaction and endothelial dysfunction. The increase of the vascular malformation rate causes significant changes in the hemodynamics of patients. However, the analysis of ultrasonic indicators of patients has a number of limitations in the diagnosis of cervical cancer (23), because benign lesions of the cervix may cause significant changes of regional blood parameters as well. Consequently, if an ultrasound is the only instrument used, it can easily result in false positives in clinical diagnoses. Thus, an analysis of the biomarkers of patients has great value in the clinical diagnosis of patients (24).

In the present study, the results of the analysis of the serological indexes of patients showed that the serum CXCL16 and E-cad levels of patients in the observation group were significantly higher than those of patients in the control group. A pairwise comparison showed that the serum CXCL16 and E-cad levels of patients increased significantly as the pathological stages progressed. The analysis also showed that E-cad is an important molecule of cell adhesion and a polar calcium-dependent transmembrane glycoprotein that maintains the intact structure of cells. Additionally, it plays an important role in the early stage of tumor development and migration. Serum CXCL16 reflects 
the level of inflammatory reaction in a local lesion area to a certain extent. As the degree of canceration increases, the serum CXCL16 level also increases. Huang et al. (15) showed that serum CXCL16 levels significantly increase as patients' degree of canceration increases. Further, ZacapalaGómez et al. (25) reported that the level of E-cad was significantly correlated with the degree of canceration in patients, which is consistent with the results of the present study.

In addition, an analysis of joint diagnostic efficacy, found that the critical values of the PSV, the RI, CXCL16, and E-cad in patients with cervical cancer were 14.25, 0.50, $40.15 \mathrm{ng} / \mathrm{mL}, 85.36 \mathrm{pmmol} / \mathrm{L}$, respectively. These results could serve as reference values for the clinical diagnosis of cervical cancer. The specificity, sensitivity and the AUC were significantly increased which indicated that the diagnostic performance was significantly improved by combining the Doppler ultrasound with serum CXCL16 and $\mathrm{E}$-cad than that of the individual parameters.

However, it should be noted that this study also had some limitations. Due to the limited sample size, the evidence level is relatively low and the conclusion of the present study is not convincing enough. Randomized controlled trials with large sample size are warranted in the future.

Overall, the results showed that the sensitivity of patients with cervical cancer in the diagnosis of color Doppler ultrasound combined with measures of serum CXCL16 and E-cad is high. Thus, it is recommended for use in clinical practice.

\section{Acknowledgments}

Funding: None.

\section{Footnote}

Reporting Checklist: The authors have completed the STARD reporting checklist. Available at http://dx.doi.org/10.21037/ tcr-21-123

Data Sharing Statement: Available at http://dx.doi. org/10.21037/tcr-21-123

Conflicts of Interest: All authors have completed the ICMJE uniform disclosure form (available at http://dx.doi. org/10.21037/tcr-21-123). The authors have no conflicts of interest to declare.
Ethical Statement: The authors are accountable for all aspects of the work in ensuring that questions related to the accuracy or integrity of any part of the work are appropriately investigated and resolved. All procedures performed in this study involving human participants were in accordance with the Declaration of Helsinki (as revised in 2013). All the patients signed the informed consent form, which was provided to and approved by the Ethics Committee of Lianyungang Maternal and Child Health Care Hospital (No.: LYG-ME2020056).

Open Access Statement: This is an Open Access article distributed in accordance with the Creative Commons Attribution-NonCommercial-NoDerivs 4.0 International License (CC BY-NC-ND 4.0), which permits the noncommercial replication and distribution of the article with the strict proviso that no changes or edits are made and the original work is properly cited (including links to both the formal publication through the relevant DOI and the license). See: https://creativecommons.org/licenses/by-nc-nd/4.0/.

\section{References}

1. Che D, Yang Z, Wei H, et al. The Adler grade by Doppler ultrasound is associated with clinical pathology of cervical cancer: Implication for clinical management. PLoS One 2020;15:e236725.

2. Smet S, Nesvacil N, Knoth J, et al. Hybrid TRUS/CT with optical tracking for target delineation in image-guided adaptive brachytherapy for cervical cancer. Strahlenther Onkol 2020;196:983-92.

3. Perniola G, Fischetti M, Tomao F, et al. Evaluation of Parametrial Status in Locally Advanced Cervical Cancer Patients after Neoadjuvant Chemotherapy: A Prospective Study on Diagnostic Accuracy of Three-Dimensional Transvaginal Ultrasound. Oncology 2020;98:603-11.

4. Wang P, Sun W, Wang L, et al. Correlations of $\mathrm{p} 53$ expression with transvaginal color Doppler ultrasound findings of cervical cancer after radiotherapy. J BUON 2018;23:769-75.

5. Sugarbaker PH. Role of carcinoembriyonic antigen assay in the management of cancer. Adv Immun Cancer Ther 1985;1:167-93.

6. Lo Gerfo P, Krupey J, Hansen HJ. Demonstration of an antigen common to several varieties of neoplasia. $\mathrm{N}$ Engl J Med 1971;285:138-41.

7. Disaia P, Morrow C, Haverback B, et al. Carcino- 
enbryonic antigen in cancer of the female reproductive system. Cancer 1977;39:2365-70.

8. Liu Z, Shi H. Prognostic Role of Squamous Cell Carcinoma Antigen in Cervical Cancer: A Meta-analysis. Dis Markers 2019;2019:6710352.

9. Darash-Yahana M, Gillespie JW, Hewitt SM, et al. The chemokine CXCL16 and its receptor, CXCR6, as markers and promoters of inflammation-associated cancers. PLoS One 2009;4:e6695.

10. Alattia JR, Tong KI, Takeichi M, et al. Cadherins. Methods Mol Biol 2002;172:199-210.

11. Li LI, Lv Y, Zhang Y, et al. Expression and clinical significance of Oct-4 and E-cad in non-small-cell lung cancer. Oncol Lett 2016;11:234-6.

12. Matloubian M, David A, Engel S, et al. A transmembrane CXC chemokine is a ligand for HIV-coreceptor Bonzo. Nat Immunol 2000;1:298-304.

13. Ke C, Ren Y, Lv L, et al. Association between CXCL16/ CXCR6 expression and the clinicopathological features of patients with non-small cell lung cancer. Oncol Lett 2017;13:4661-8.

14. Peng J, Qi S, Wang P, et, al. Meta-analysis of downregulated E-cad as a poor prognostic biomarker for cervical cancer. Future Oncol 2016;12:715-26.

15. Huang Y, Zhang J, Cui ZM, et al. Expression of the CXCL12/CXCR4 and CXCL16/CXCR6 axes in cervical intraepithelial neoplasia and cervical cancer. Chin J Cancer 2013;32:289-96.

16. Cohen JF, Korevaar DA, Altman DG, et al: STARD 2015 guidelines for reporting diagnostic accuracy studies: explanation and elaboration. BMJ Open 2016;6:e12799.

17. Amant F, Mirza MR, Koskas M, et al. Cancer of the corpus uteri. Int J Gynaecol Obstet 2018;143:37-50.

18. Bell L, Holloway L, Bruheim K, et al. Dose planning variations related to delineation variations in MRIguided brachytherapy for locally advanced cervical cancer. Brachytherapy 2020;19:146-53.

Cite this article as: Wang W, Gao X, Zhu Y, Qi Y, Wang Y. Diagnostic significance of a color Doppler ultrasound combined with serum CXCL16 and E-cad in cervical cancer. Transl Cancer Res 2021;10(3):1492-1499. doi: 10.21037/tcr-21-123
19. Liang H, Fu M, Liu FM, et al. Transvaginal threedimensional color power Doppler ultrasound and cervical MVD measurement in the detection of cervical intraepithelial neoplasia. Eur Rev Med Pharmacol Sci 2014;18:1979-84.

20. Dodampahala SH, Jayakody SN, Gunathilake WCCC, et al. Transvaginal Color Doppler in the Assessment of Cervical Carcinoma and Pre-Cancer: Evidence from a Case Control Study Using Colour Doppler Ultrasonography Pulsatility Index of Uterine Vasculature. Advances in Reproductive Sciences 2016;4:93-9.

21. Seppenwoolde Y, Assenholt MS, Georg D, et al. Importance of training in external beam treatment planning for locally advanced cervix cancer: Report from the EMBRACE II dummy run. Radiother Oncol 2019;133:149-55.

22. Berger T, Seppenwoolde Y, Pötter R, et al. Importance of Technique, Target Selection, Contouring, Dose Prescription, and Dose-Planning in External Beam Radiation Therapy for Cervical Cancer: Evolution of Practice From EMBRACE-I to II. Int J Radiat Oncol Biol Phys 2019;104:885-94.

23. Daskalakis G, Diamantopoulos D, Theodora M, et al. $3 \mathrm{D}$ vocal power Doppler sonography for the estimation of tumor volume and vascularization in stage IB1 cervical cancer. Arch Gynecol Obstet 2018;298:617-22.

24. Furukawa N, Kawaguchi R, Kajihara H, et al. Evaluation of the vessels of the cardinal ligament by transrectal ultrasonography with color Doppler imaging. J Clin Ultrasound 2011;39:502-5.

25. Zacapala-Gómez AE, Navarro-Tito N, Alarcón-Romero LDC, et al. Ezrin and E-cad expression profile in cervical cytology: a prognostic marker for tumor progression in cervical cancer. BMC Cancer 2018;18:349.

(English Language Editor: L. Huleatt) 